\title{
A Rapid Method for Detecting and Quantifying Bacterial DNA in Rust Fungal DNA Samples
}

\author{
C. W. Barnes and L. J. Szabo
}

U.S. Department of Agriculture-Agricultural Research Service, Cereal Disease Laboratory, Department of Plant Pathology, University of Minnesota, St. Paul.

Accepted for publication 7 September 2007.

\begin{abstract}
Barnes, C. W., and Szabo, L. J. 2008. A rapid method for detecting and quantifying bacterial DNA in rust fungal DNA samples. Phytopathology 98:115-119.

Bacterial DNA contamination of rust fungal DNA can be a significant problem for sequencing the rust fungus. Sequence assembly is much more difficult if the sequence contigs are mixed with bacterial sequence. A quantitative real-time polymerase chain reaction (qPCR) assay was developed to quantify bacterial DNA within rust fungal DNA samples and the results were compared with those obtained from traditional CFU counts. Real-time PCR showed higher values of DNA contamination than CFU. However, the ranking of samples from low to high for bacterial

tested by adding known quantities of Escherichia coli DNA to Puccinia graminis DNA samples. The assay reliably quantified bacterial contamination at $\geq 1.0 \%$ of the total sample DNA. When bacterial contamination was $<1.0 \%$, fungal DNA also occasionally was amplified, nullifying the quantification measurement. However, primer specificity was not simply the product of the ratio of bacterial DNA to fungal DNA. Bacterial contamination could be quantified below $1.0 \%$ if the bacterial DNA concentration was $\approx 70 \mathrm{pg} / \mu \mathrm{l}$ or greater. Therefore, spiking the fungal samples with a known concentration of $E$. coli bacterial DNA successfully eliminated the amplification of fungal DNA, making quantification of contaminating bacterial DNA possible for samples with low contamination levels.
\end{abstract} contamination was consistent between the methods. Reasons for the differences between the methods are discussed. The qPCR assay was
Additional keywords: spore mats, SYBR Green I.
Bacterial contamination is a significant concern when utilizing molecular methods, particularly amplified fragment length polymorphism, random amplified polymorphic DNA, and DNA sequencing, for the study of nonbacterial plant pathogens. Obligate pathogens, such as rust fungi, are particularly problematic because they cannot be purified in culture. Application of antibiotics to reduce bacterial contamination is common but direct methods for measuring the bacterial DNA levels are currently unavailable. Colony counting from plates can be useful but is time consuming and may misrepresent total bacterial DNA of a given sample due to the fact that some bacteria are not culturable in a given medium $(19,20)$, or due to media dependent growth rates or dead cells. For DNA sequencing, detection of sequence error (13) and contamination by DNA from other organisms (21) has been done by the use of computational tools or BLAST search databases for known bacterial sequence (2), but these methods are performed after sequence assembly. A direct measurement of bacterial DNA would allow contaminated samples to be eliminated prior to sequencing and thereby reduce the time and effort in sequence assembly. Real-time or quantitative PCR (qPCR) offers an easy and reproducible method for determining bacterial DNA levels in fungal DNA samples destined to be sequenced. The use of the qPCR method to quantify bacterial DNA is advantageous in determining the detection limits rather than just a positive or negative assay.

Conserved regions of the $16 \mathrm{~S}$ rDNA of bacteria commonly are used to develop primer pairs to co-amplify many bacterial species. Various qPCR assays have been developed using these

Corresponding author: C. W. Barnes; E-mail address: barn0107@umn.edu

doi:10.1094/PHYTO-98-1-0115

This article is in the public domain and not copyrightable. It may be freely reprinted with customary crediting of the source. The American Phytopathological Society, 2008. types of inclusive primers in the $16 \mathrm{~S}$ rDNA to study populations of bacteria in the human intestinal tract (12) and oral cavity (15), but may not be applicable in other environments. However, a "universal" eubacterial primer pair was developed by Schwieger and Tebbe (17) and verified by Schmalenberger et al. (16) using bacteria collected from the rhizosphere of plants collected in agricultural fields. Subsequently, Labrenz et al. (9) created a qPCR assay using the primer pair developed by Schwieger and Tebbe (17) to quantify total bacteria from Baltic Sea water samples.

The aim of this study was to develop a qPCR assay to detect and quantify the relative amounts of bacterial DNA within fungal DNA samples prior to sequencing the genome of the rust fungus Puccinia graminis f. sp. tritici. The qPCR assay was compared with the traditional CFU count method to evaluate the accuracy and benefits of the qPCR assay. Finally, the qPCR assay was used to examine the variation in bacterial DNA levels in rust fungal DNA samples obtained from different sources such as leaf tissue, dried spores, or germinated spores mats. The primer pair developed by Schwieger and Tebbe (17) was used to quantify bacterial DNA because it will amplify DNA from a wide diversity of bacteria and because of its use in several studies investigating bacterial communities. The intercalating dye SYBR Green I was used to detect the possible diverse PCR products.

\section{MATERIALS AND METHODS}

DNA extraction. $P$. graminis f. sp. tritici (CDL 59KS19, race MCCF; CDL 78-21-BB463, race DBFJ) was used for genomic sequencing and was increased on the wheat cv. McNair 701 as described by Liu et al. (10) in a growth chamber. Freshly collected spores $(0.2 \mathrm{~g})$ were germinated overnight in sterilized glass baking dishes containing germination solution (10) prepared with sterile water. Germinated urediniospores (mats) were harvested and filtered to remove residual germination solution. Spore mats 
were washed by vortexing with $2 \mathrm{ml}$ of sterile water. $P$. graminis DNA was extracted from spore mats using the OmniPrep Genomic DNA kit (GenoTech Inc., St. Louis).

To evaluate different sources of rust fungal DNA for bacterial DNA contamination, rust fungal DNA was extracted from either dried ungerminated urediniospores (1 to $20 \mathrm{mg}$ ), diced infected leaf tissue with visible uredinia (20 to $30 \mathrm{mg}$ ), or germinated mats as described above, but in a smaller volume. All samples were shaken in 2-ml tubes with 1-mm glass beads (Lysing Matrix C; Bio 101, Carlsbad, CA) and $25 \mathrm{mg}$ of diatomaceous earth (SigmaAldrich, St. Louis) in a FastPrep shaker FP120A (MP Biomedicals, Solon, $\mathrm{OH}$ ) for $10 \mathrm{~s}$ at a speed setting of 5 (1) and extracted with the OmniPrep Genomic DNA kit (GenoTech Inc.). Rust fungal DNA sources were $P$. striiformis (dried urediniospores), $P$. coronata, $P$. graminis (infected leaf tissue), and $P$. triticina (germinated spore mats). Uninfected Triticum aestivum (leaf tissue) was included as a control.

Genomic DNA of Escherichia coli K-12 strain MG1655 was provided by B. Martinez-Vaz, University of Minnesota. This single source of bacterial DNA was used as a standard throughout the study. All DNA concentrations were determined fluorometrically using a DyNA Quant200 Fluorometer (Hoefer, Inc., San Francisco).

qPCR protocol and analysis. The universal bacterial primer pair used in this study, Com1 (dCAGCAGCCGCGGTAATAC) and Com 2 (dCCGTCAATTCCTTTGAGTTT), amplifies the variable regions V4 and V5 of the bacterial 16S rRNA gene, and produces amplification products of $\approx 400 \mathrm{bp}$ (17). In this study, an unphosphorylated Com2 was used rather than a $5^{\prime}$ phosphorylated Com2 as reported by Schwieger and Tebbe (17).

Bacterial DNA was quantified with a SmartCycler qPCR device (Cepheid, Sunnyvale, CA) using the SYBR Green I fluorescent dye detection system. Reactions were performed in a $25-\mu$ l final volume containing $2 \mu \mathrm{l}$ of sample DNA and $23 \mu \mathrm{l}$ of master mix containing $1 \times$ FastStart master mix (LightCycler FastStart DNA Master SYBR Green I; Roche, Basel, Switzerland) and $1.0 \mu \mathrm{M}$ each primer (Smart Notes from Cepheid).

Amplification conditions consisted of initial denaturation at $95^{\circ} \mathrm{C}$ for $10 \mathrm{~m}$ followed by 45 three-step cycles of $95^{\circ} \mathrm{C}$ for $15 \mathrm{~s}$, $60^{\circ} \mathrm{C}$ for $30 \mathrm{~s}$, and $72^{\circ} \mathrm{C}$ for $30 \mathrm{~s}$. The threshold line was set at 30 as an arbitrarily chosen point within the log-linear phase of amplification, significantly greater than the variability in background fluorescence and well before the reaction plateau (3). After completion of the PCR, a melting curve was obtained by heating the amplicon from 60 to $95^{\circ} \mathrm{C}$ at a rate of $0.2^{\circ} \mathrm{C} / \mathrm{s}$. In order to rule out false positives, melting curve analysis and verifying the size of the amplicon on $1.0 \%$ agarose gels was done for each experiment. All experiments included negative (no target DNA) and serial dilutions of an E. coli DNA standard control.

Absolute quantification of DNA in qPCR was accomplished using standard curves of known concentrations of DNA. In this study, standard curves were generated using $1: 10^{\mathrm{x}}$ serial dilutions of $E$. coli (MG1655) over four orders of magnitude and plotted as the log of DNA concentration verses the threshold cycle $(\mathrm{Ct})$ value generated by the second derivative. The $\mathrm{Ct}$ value of the second derivative is the cycle at which the greatest increase in fluorescence has occurred and represents the log-linear phase of the amplification growth curve. PCR efficiency was calculated as $\mathrm{E}=\left(10^{\text {(-slope) }}-1\right) \times 100$ (with the $\mathrm{Ct}$ value on the $\mathrm{x}$-axis) for each assay. Assays with $\mathrm{E}<90 \%$ and $r^{2}<0.99$ were not analyzed and were repeated.

Identifying the amplicon. As expected from bacterial sequence data, the Com1/Com2 primer pair produced a 400-bp fragment during the qPCR. However, qPCR assays of $P$. graminis DNA samples spiked with very low concentrations of $E$. coli DNA occasionally produced a second product of $600 \mathrm{bp}$. The identity of the 400- and 600-bp amplification products was determined by excising each band from an agarose gel, purifying the DNA using the Wizard SV Gel and PCR Clean-Up System (Promega Corp., Madison, WI), and sequencing the DNA fragments. The excised DNA fragments were PCR amplified in a 50- $\mu$ l reaction mixture as described in Anikster et al. (1). DNA sequencing reactions were preformed using a Thermo Sequencase Prime Cycle sequencing kit (Amersham Biosciences, Piscataway, NJ) and analyzed on an automated DNA sequencer (Li-Cor, Lincoln, NE). Three clones were sequenced for each sample, and the DNA sequence data was assembled and edited with Sequencer (Genecodes, Ann Arbor, MI). Consensus sequences were compared with other DNA sequences through BLASTN on the National Center for Biotechnology Information website. Specific sequence comparisons were made between the excised amplicons and other fungi of varying relatedness to $P$. graminis to pinpoint sequence differences and determine whether the amplicon was derived from $P$. graminis by aligning sequences using MacVector (MacVector, Inc., Cary, NC). The DNA sequences, with GenBank accession numbers, used for the comparisons were three rust fungal species (P. graminis f. sp. tritici, AY125409; P. pelargoniizonalis, AY123316; and Uromyces appendiculatus, AY123307), two distantly related rust fungal species (Agaricostibum hyphaenes, AHU0809 and Bensingtonia subrosea, BENJCM5735), two Ascomycetes (Magnaporthe grisea, AB026819.1 and Aspergillus spp., AB179824.1), and E. coli K12 (U00096). The above DNA sequences were further analyzed graphically in PAUP 4.0b5 using Neighbor-joining analysis with 1,000 bootstrap replicates (18).

Spiking experiments. To determine whether bacterial DNA could be detected in a $P$. graminis DNA sample, a sample of bacteria-free $P$. graminis was spiked with $E$. coli DNA equal to 0 , $0.01,0.1,1.0$, and $10 \%$ of the total DNA, equivalent to $0,2,20$, and $200 \mathrm{pg}$ and $2.0 \mathrm{ng}$ of E. coli DNA, respectively. The same $E$. coli DNA concentrations were used to generate the accompanying standard curve used to calculate bacterial DNA concentrations.

The same spiking protocol also was used to determine whether the assay could quantify total bacterial DNA from a mixture of more than one bacterial species. The source of bacterial DNA species in the mixture was assumed to be from the leaf tissue from which the $P$. graminis DNA was extracted and the addition of $E$. coli DNA spiked into the sample. The underlying bacterial DNA contamination was quantified by subtracting the concentration of the added $E$. coli DNA from the total bacterial DNA determined by the qPCR assay.

Precision of the qPCR assay was evaluated using a single sample of $P$. graminis assayed in triplicate. A single DNA extraction of $P$. graminis from leaf tissue was performed using the OmniPrep Genomic DNA kit (GenoTech Inc.) and aliquoted into three separate microfuge tubes. Individual qPCR assays were performed on each aliquot on separate days.

Comparing qPCR and CFU analyzes. Eight independent DNA extractions of $P$. graminis were analyzed for bacterial contamination using both the qPCR and CFU methods. The qPCR method was used as described above on undiluted DNA and a 1:10 DNA dilution in sterile water and averaged. If a fungal band was observed on the accompanying agarose gel, an aliquot of the sample was spiked with $E$ coli DNA and the underlying bacterial DNA concentration determined by subtracting the amount of spiked DNA. The CFU method was done using serial dilutions from $10^{0}$ to $10^{2}$ made in sterile water. Each dilution $(50 \mu \mathrm{l})$ was spread onto Luria-Bertani agar plates and grown overnight at $37^{\circ} \mathrm{C}$. Colonies on each plate were counted and CFU per milliliter for each sample was determined. Each experiment was repeated three times and averaged. Level of bacterial contamination was expressed as percentage of bacterial CFU relative to the total number of fungal spores, using the estimate of 450,000 urediniospores per milligram of spores (14).

Evaluating DNA sources. Laboratory DNA samples from $P$. coronata, $P$. graminis (from infected leaf tissue), $P$. striiformis 
(from dried urediniospores), P. triticina (from germinated spore mats), and T. aestivum DNA were analyzed to examine bacterial DNA contamination differences between tissue preparation protocols. DNA extraction of each sample was described above. The qPCR method was used as described above on undiluted DNA and a 1:10 DNA dilution in sterile water and averaged.

\section{RESULTS}

Identifying the amplicon. Evaluating the primer pair. Use of the Com 1 and Com 2 primer pair in the qPCR assay occasionally resulted in two amplicons. The sequence alignment of the Com1 primer with other fungi and $E$. coli rDNA showed one mismatch with the $18 \mathrm{~S}$ rDNA sequence of $P$. graminis and other fungi (data not shown). Similarly, sequence of the Com 2 primer showed only 1 mismatch between Basidiomycetes fungi and two mismatches to the Ascomycetes fungi used in this analysis (data not shown). These results substantiate the idea that, although the Com 1 and Com2 primer pair were useful in amplifying bacterial DNA, they also may amplify fungal DNA. However, amplification of fungal DNA was easily discernable on an agarose gel due to amplicon size differences (Fig. 1).

Examining the $P C R$ products. In samples with both $P$. graminis DNA and bacterial DNA, two bands of $\approx 600$ and 400 bp in length occasionally were observed (Fig. 1). Excising each band from the agarose gel and sequencing the fragment BLASTN results indicated that bands of $\approx 600 \mathrm{bp}$ were of fungal origin (18S rDNA), whereas those $\approx 400$ bp in size were derived from bacteria (16S rDNA). Sequence analysis of the 600-bp band confirmed that the source of DNA was from $P$. graminis, and not another fungal contaminant or bacteria, by inspection of the resulting phylogenetic tree (data not shown). Excluding primer sequence differences, the sequence of the 600-bp amplicon excised from the agarose gel

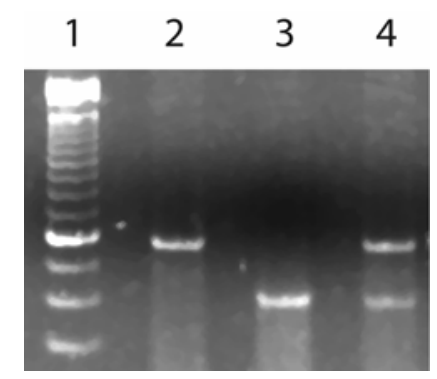

Fig. 1. A $1.0 \%$ agarose gel of real-time polymerase chain reaction amplification of Puccinia graminis DNA showing two amplicons generated using primers Com1 and Com2. The molecular size marker in lane 1 is a 100-bp ladder. Lane 2 contains $P$. graminis DNA free of bacterial contamination, whereas lane 3 contains the same $P$. graminis DNA sample spiked with $1.0 \%$ Escherichia coli DNA. Sizes of the amplicons are approximately 600 and $400 \mathrm{bp}$ and occasionally are co-amplified (lane 4$)$. Lane 4 contains P. graminis DNA spiked with $0.1 \%$ E. coli DNA.

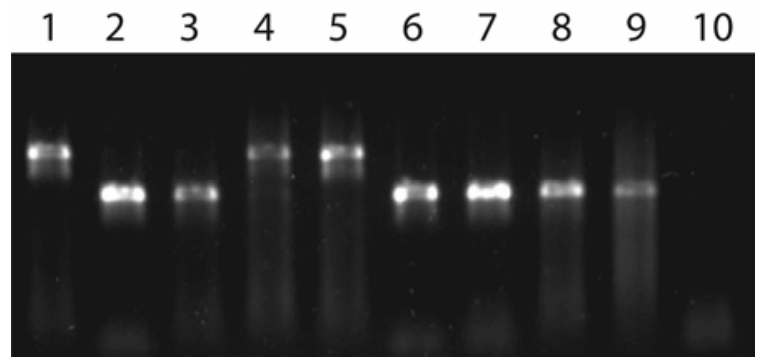

Fig. 2. A $1.0 \%$ agarose gel of real-time polymerase chain reaction amplification of Puccinia graminis DNA using primers Com1 and Com2 spiked with Escherichia coli DNA. Lanes 1 through 5 are $P$. graminis DNA spiked with 0, $10,1,0.1$, and $0.01 \%$ E. coli DNA. Lanes 6 through 10 are DNA standards of $2 \mathrm{ng}$ and 200, 20, 2, and $0 \mathrm{pg}$ of E. coli DNA. was $99.5 \%$ similar to the available 560-bp P. graminis $\mathrm{f}$. sp. tritici $18 \mathrm{~S}$ rDNA sequence. These results suggest that the Com1 and Com2 primer pair amplified $P$. graminis DNA and not a second region, different-sized 16S rDNA of bacterial DNA, or other contaminant DNA.

Spiking experiments. Spiking of bacteria-free $P$. graminis DNA with known concentrations of $E$. coli DNA was used to demonstrate the effectiveness of the assay. Spiking bacteria-free $P$. graminis DNA with $1.0 \%$ E. coli DNA (200 pg) approximates the threshold at which only bacterial DNA was amplified, whereas spiking with $0.1 \%$ or less of E. coli DNA $(\leq 20 \mathrm{pg})$ often resulted in two bands or just the 600-bp band (Fig. 2). The mismatch of the Com 1 primer one base from the $3^{\prime}$ terminal end, along with the longer 600-bp size of the amplicon, likely resulted in less efficient amplification of fungal DNA compared with bacterial DNA.

A second sample of $P$. graminis DNA, extracted from leaf tissue, was spiked with a serial dilution of genomic E. coli DNA to demonstrate that the qPCR assay could detect and quantify mixtures of DNA from different bacterial species. At each level of added $E$. coli DNA, the qPCR detected the amount of $E$. coli DNA added to the reaction plus bacterial DNA already present in the sample (Table 1). Variation between dilutions in the final DNA contamination of the original sample was in the tens of picograms. No fungal band was observed in the accompanying agarose gel in any part of this series of reactions likely due to the underlying bacterial DNA contamination of the original sample at $\approx 128 \mathrm{pg} / \mu \mathrm{l}$ (average of Table 1). Total DNA concentration of the sample was $40 \mathrm{ng} / \mu \mathrm{l}$, giving a range of 0.2 to $0.4 \%$ bacterial DNA contamination using the minimum and maximum of 95 and $165 \mathrm{pg} / \mu \mathrm{l}$ from Table 1 , respectively.

Assay precision is essential if the qPCR technique is to be applicable for determining bacterial DNA contamination in rust DNA samples. Therefore, a single sample of $P$. graminis was assayed with the bacterial primer pair in triplicate on separate days. The contamination level of the original sample was $\approx 3.0 \%$, with $\mathrm{Ct}$ values among the three assays varying by $0.4 \%$ (Table 2 ).

Comparing qPCR and CFU analysis. Comparing the use of the qPCR assay with the CFU count method for estimating bacterial DNA contamination was done with eight independent DNA

TABLE 1. Escherichia coli DNA added to a Puccinia graminis DNA sample known to be contaminated with bacterial DNA

\begin{tabular}{lcc}
\hline \multicolumn{3}{c}{ Amount $(\mathrm{pg} / \mu \mathrm{l})$} \\
\hline $\begin{array}{l}\text { Quantity of } \\
\text { E. coli } \text { DNA added }\end{array}$ & $\begin{array}{c}\text { Quantity determined } \\
\text { by qPCR }^{\mathrm{a}}\end{array}$ & $\begin{array}{c}\text { Determined level of } \\
\text { contamination }^{\text {b }}\end{array}$ \\
\hline 0 & 165 & 165 \\
2 & 126 & 124 \\
20 & 154 & 134 \\
200 & 295 & 95 \\
2,000 & 2,124 & 124 \\
\hline
\end{tabular}

${ }^{\mathrm{a}} \mathrm{qPCR}=$ quantitative real-time polymerase chain reaction.

${ }^{\mathrm{b}}$ Calculated by subtracting the quantify of $E$. coli DNA added from the quantity determined by qPCR.

Table 2. Puccinia graminis DNA sample assayed in triplicate for bacterial DNA

\begin{tabular}{|c|c|c|c|}
\hline Sample, rep ${ }^{\mathrm{a}}$ & $\mathrm{Ct}$ value ${ }^{\mathrm{b}}$ & Concentration $(n g / \mu l)^{\mathrm{c}}$ & Contamination $(\%)$ \\
\hline \multicolumn{4}{|l|}{$28 \mathrm{ng} / \mu \mathrm{l}$} \\
\hline 1 & 15.73 & 0.89 & 3.2 \\
\hline 2 & 15.8 & 0.83 & 3 \\
\hline 3 & 15.94 & 0.77 & 2.8 \\
\hline
\end{tabular}

a Total DNA concentration determined using a DyNA Quant200 Fluorometer (Hoefer). Quantitative real-time polymerase chain reaction (qPCR) assay replications were performed on separate days.

${ }^{\mathrm{b}} \mathrm{Ct}=$ threshold cycle.

c Bacterial DNA concentration determined using the qPCR assay described in this study. 
extractions of $P$. graminis. Bacterial contamination determined by qPCR varied by two orders of magnitude, whereas CFU count analysis varied by three orders of magnitude among samples (Table 3). The fungal band at $600 \mathrm{bp}$ was not observed among these assays, which suggests that lower limits of detection of absolute levels of bacterial DNA concentrations were $\geq 70 \mathrm{pg} / \mu \mathrm{l}$. Estimates of bacterial contamination by CFU counts were somewhat consistent with those obtained by qPCR in that they varied significantly among the $P$. graminis samples and ranked from lowest to highest contamination similarly (Table 3).

Evaluating DNA sources. DNA extractions were performed either on dried spores or from germinated mats for $P$. striiformis and $P$. triticina, respectively. DNA from $P$. coronata, collected from Bromus inermis (smooth brome grass), and DNA from wheat plant tissue samples was extracted from ground leaf tissue. Contamination levels varied, with leaf tissue extractions having the highest levels of contamination and extractions from dried spores having the least (Table 4). Bacterial DNA concentration of $90 \mathrm{pg} / \mu \mathrm{l}$ was considerably lower for $P$. striiformis compared with other samples but within the lower limits of bacterial DNA detected among samples in Table 3.

\section{DISCUSSION}

The primary objective of this study was to develop a fast and reliable qPCR method to evaluate $P$. graminis DNA samples destined for sequencing for bacterial DNA contamination. A clear advantage of the qPCR method is that it was a direct measure of contaminant DNA compared with the CFU method that measures living cells. The Com1/Com2 "universal" primer pair was used because it amplified DNA from a wide range of bacteria (17) and it had been used previously in a variety of applications, including quantifying bacteria in the rhizosphere (16) and in seawater (9).

TABLE 3. Comparison of the quantitative real-time polymerase chain reaction (qPCR) assay to the CFU count method for determining bacterial DNA contamination in Puccinia graminis DNA samples

\begin{tabular}{lcccc}
\hline & & \multicolumn{3}{c}{ Contamination } \\
\cline { 3 - 5 } Sample $(\mathrm{ng} / \mu \mathrm{l})^{\mathrm{a}}$ & Conc. $(\mathrm{ng} / \mu \mathrm{l})^{\mathrm{b}}$ & $\mathrm{qPCR}(\%)$ & $\mathrm{CFU}(\%)^{\mathrm{c}}$ & Rank $^{\mathrm{d}}$ \\
\hline 73 & 0.08 & 0.11 & 0 & $1-1$ \\
56 & 0.07 & 0.13 & 0.0001 & $2-2$ \\
58 & 0.15 & 0.26 & 0.0001 & $3-2$ \\
61 & 0.4 & 0.66 & 0.002 & $4-4$ \\
70 & 1.2 & 1.7 & 0.4 & $5-8$ \\
66 & 3.1 & 4.7 & 0.2 & $6-6$ \\
50 & 4.6 & 9.2 & 0.2 & $7-6$ \\
62 & 6.4 & 10.3 & 0.1 & $8-5$ \\
\hline
\end{tabular}

${ }^{a}$ Total DNA concentration determined using a DyNA Quant200 Fluorometer (Hoefer).

${ }^{\mathrm{b}}$ Bacterial DNA concentration determined using the qPCR assay described in this study.

${ }^{c}$ Percent contamination is expressed as the percentage of bacterial CFU relative to the total number of fungal spores on the estimate of 450,000 urediniospores per milligram of spores (14).

${ }^{\mathrm{d}}$ Contamination rank. The eight samples were ranked 1 to 8 , from the lowest contamination level to the highest. The number on the left is the qPCR ranking, the number on the right is the CFU ranking. CFU counts with the same percent contamination were assigned the same ranking.
The qPCR results indicated that the assay worked consistently for quantifying bacterial contamination levels of $\geq 1.0 \%$ total DNA in $P$. graminis DNA samples, and as low as $0.1 \%$. The assay occasionally would amplify both the fungal and bacterial DNA if the sample was contaminated with $<1.0 \%$ bacterial DNA. Low levels of nonspecific amplification are possible when using a broadspectrum assay for a sample that is predominately nontarget DNA. However, even when both fungal and bacterial DNA was co-amplified, observation of the 400-bp band on an agarose gel verified the presence of bacterial DNA in the sample. Limits of primer specificity appear to be influenced mostly by total bacterial DNA. For samples with high fungal DNA concentrations, percentages of $<1.0 \%$ of bacterial DNA were quantified consistently, with the limit of bacterial DNA being $\approx 70 \mathrm{pg} / \mu \mathrm{l}$, below which fungal and bacterial DNA both were amplified. The addition of a known quantity of bacterial DNA to fungal DNA samples with low DNA concentration proved to be effective in quantifying even lower levels of bacterial DNA contamination and eliminating nonspecific amplification of fungal DNA.

Significant differences were found between the qPCR and the CFU method in quantifying bacterial DNA. Similar discrepancies between PCR and culturing methods have been found by other authors $(4,8,11)$ and may result from a low estimate of DNA in the CFU method from unculturable or slow-growing bacteria, nonviable cells, or free DNA. Specific to this study, the standardization of CFUs to spore counts (per milligram of spores) to obtain a contamination percentage may affect the difference between CFU counts and qPCR results. Furthermore, the variation in the multinumber of $16 \mathrm{~S}$ rRNA gene copies $(5,11)$ compared with the $E$. coli DNA standard may inflate the value of bacterial DNA by the qPCR method, thus further increasing the variation between the two methods. However, the ranking of samples was in close agreement, indicting that viable culturable bacterial cells were a significant component of the contamination in this study. Other studies comparing CFU and qPCR for quantifying bacteria have found closer agreement between the methods $(6,7)$. However, these studies used primers specific to particular bacterial families, thus reducing the interaction of the primers with nontarget DNA and reducing variation in 16S rRNA gene copy numbers. Our goal was to quantify all bacterial DNA present in a given fungal sample. Therefore, the use of more specific primers was not warranted.

The assay described in this study also was used to evaluate bacterial DNA contamination from different sources of rust fungal DNA. As expected, fungal DNA from infected leaf tissue had higher bacterial contamination levels compared with germinated spore mats or lyophilized spores. For our purpose of sequencing, maximizing the $P$. graminis DNA concentration was the priority. Lyophilized spores were not a good source of DNA due to the reduced DNA extraction efficiency, and leaf tissue had relatively high bacterial contamination levels. Therefore, spore mats were the best option. To minimize contamination in spore mats, we applied antibiotics to the germination solution. However, the effectiveness of the antibiotics varied among samples (data not shown), and the assay proved to be useful in detecting samples with relatively high contamination levels among different mats.

The qPCR assay we developed functioned well for our purposes by reducing the time needed to estimate bacterial DNA contami-

TABLE 4. Bacterial DNA contamination of rust fungal DNA from varying sources and wheat DNA

\begin{tabular}{lcccc}
\hline Sample & Total DNA $(\mathrm{ng} / \mu \mathrm{l})^{\mathrm{a}}$ & ${\text { Concentration }(\mathrm{ng} / \mu \mathrm{l})^{\mathrm{b}}}^{\mathrm{a}}$ & Contamination $(\%)$ & DNA source \\
\hline Puccinia coronata & 360 & 8 & 2.2 & Leaf tissue \\
$P$. striiformis & 20 & 0.09 & 0.45 & Lyophilized spores \\
$P$. triticina & 90 & 0.97 & 1.1 & Germinated mats \\
Triticum aestivum & 300 & 23 & 7.67 & Leaf tissue \\
\hline
\end{tabular}

a Total DNA concentration determined using a DyNA Quant200 Fluorometer (Hoefer).

${ }^{\mathrm{b}}$ Bacterial DNA concentration determined using the QPCR assay described in this study. 
nation prior to sequencing. Quantification of bacterial DNA in fungal DNA samples was performed in a single day compared with the 5 to 6 days required for CFU counts. The addition of a "universal" probe for bacterial DNA $(11,12)$ could increase sensitivity, but it may make the assay more selective and increase the cost.

\section{ACKNOWLEDGMENTS}

Mention of a trademark name or proprietary product does not constitute a guarantee by the United States Department of Agriculture or the University of Minnesota. We thank J. L. Johnson for his technical assistance and D. Malvick and M. Ordóñez for critical comments on the manuscript.

\section{LITERATURE CITED}

1. Anikster, Y., Szabo, L. J., Eilam, T., Manisterski, J., Koike, S. T., and Bushnell, W. R. 2004. Morphology, life cycle biology, and DNA sequence analysis of rust on garlic and chives from California. Phytopathology 94:569-577.

2. Astua-Monge, G., Lyznik, A., Jones, V., Mackenzie, S. A., and Vallejos, C. E. 2002. Evidence for a prokaryotic insertion-sequence contamination in eukaryotic sequences registered in different databases. Theor. Appl. Genet. 104:48-53.

3. Bustin, S. A. 2002. Quantification of mRNA using real-time reverse transcription PCR (RT-PCR): Trends and problems. J. Mol. Endocrinol. 29:23-39.

4. Castillo, M., Martín-Orúe, S. M., Manzanilla, E. G., Badiola, I., Martín, M., and Gasa, J. 2006. Quantification of total bacteria, enterobacteria and lactobacilli populations in pig digesta by real-time PCR. Vet. Microbiol. 114:165-170

5. Farelly, V., Rainly, F. A., and Stackebrandt, E. 1995. Effect of genome size and $r r n$ gene copy number on PCR amplification of 16S rRNA genes from a mixture of bacterial species. Appl. Environ. Microbiol. 61:27982801.

6. Fu, C. J., Carter, J. N., Li, Y., Porter, J. H., and Kerley, M. S. 2006. Comparison of agar plate and real-time PCR on enumeration of Lactobacillus, Clostridium perfringens and total anaerobic bacteria in dog faeces. Lett. Appl. Microbiol. 42:490-494.

7. Furet, J. P., Quenee, P., and Tailliez, P. 2002. Identification and quantification of lactic acid bacteria by real time quantitative PCR assay. Sci. Aliment. 22:33-44.
8. Huijsdens, X. W., Linskens, R. K., Mak, M., Meuwissen, S. G. M., Vandenbroucke-Grauls, C. M. J. E., and Savelkoul, P. H. M. 2002. Quantification of bacteria adherent to gastrointestinal mucosa by realtime PCR. J. Clin. Microbiol. 40:4423-4427.

9. Labrenz, M., Brettar, I., Christen, R., Flavier, S., Botel, J., and Hofle, M. G. 2004. Development and application of a real-time PCR approach for quantification of uncultured bacteria in the central Baltic Sea. Appl. Environ. Microbiol. 70:4971-4979.

10. Liu, Z., Szabo, L. J., and Bushnell, W. R. 1993. Molecular cloning and analysis of abundant and stage-specific mRNAs from Puccinia graminis. Mol. Plant-Microbe Interact. 6:84-91.

11. Nadkarni, M. A., Martin, F. E., Jacques, N. A., and Hunter, N. 2002. Determination of bacterial load by real-time PCR using a broad-range (universal) probe and primers set. Microbiology 148:257-266.

12. Ott, S.J., Musfeldt, M., Ullmann, U., Hampe, J., and Schreiber, S. 2004 Quantification of intestinal bacterial populations by real-time PCR with a universal primer set and minor groove binder probes: A global approach to the enteric flora. J. Clin. Microbiol. 42:2566-2572.

13. Richterich, P. 1998. Estimation of errors in "raw" DNA sequences: A validation study. Genome Res. 8:251-259.

14. Rowell, J. B. 1984. Controlled infection by Puccinia graminis f. sp. tritici under artificial conditions. Pages 291-332 in: The Cereal Rusts, vol. 1. Academic Press, New York.

15. Sakamoto, M., Huang, Y., Ohnishi, M., Umeda, M., Ishikawa, I., and Benno, Y. 2004. Changes in oral microbial profiles after periodontal treatment as determined by molecular analysis of 16S rRNA genes. J. Med. Microbiol. 53:563-571.

16. Schmalenberger, A., Schwieger, F., and Tebbe, C. C. 2001. Effect of primers hybridizing to different evolutionarily conserved regions of the small-subunit rRNA gene in PCR-based microbial community analysis and genetic profiling. Appl. Environ. Microbiol. 67:3557-3563.

17. Schwieger, F., and Tebbe, C. C. 1998. A new approach to utilize PCRsingle-strand-conformation for 16S rRNA gene-based microbial community analysis. Appl. Environ. Microbiol. 64:4870-4876.

18. Swofford, D. L. 2001. PAUP*. Phylogenetics Analysis Using Parsimony (*and other methods). Sinauer Associates, Sunderland, MA.

19. Wade, W. 2002. Unculturable bacteria-The uncharacterized organisms that cause oral infections. J. R. Soc. Med. 95:81-83.

20. Ward, D. M., Weller, R., and Bateson, M. M. 1990. 16S rRNA sequences reveal numerous uncultured microorganisms in a natural community. Nature 345:63-65.

21. White, O., Dunning, T., Sutton, G., Adams, M., Venter, J. C., and Fields, C. 1993. A quality control algorithm for DNA sequencing projects. Nucleic Acids Res. 21:3829-3838. 